\title{
Development of the Motor System: Effects of Radiation on Developing Corticospinal Neurons and Locomotor Function
}

\author{
Constance J. D'Amato and Samuel P. Hicks ${ }^{1}$ \\ Department of Pathology, University of Michigan Medical Center, \\ Ann Arbor, Michigan 48109
}

Received March 6, 1980

Corticospinal (CS) neurons demonstrated by retrograde axonal HRP from the cord in adult rats were concentrated in a minor rostral band in area 10, projecting to the cervical cord, and a major caudal band, areas 3, 4, and 6, projecting as far as lumbar levels. The gap between the bands contained CS neurons projecting to the cervical cord only until age 2 weeks, but if the caudal band was ablated, the projections persisted. The problems investigated were (i) How would CS neurons develop postnatally and be distributed in rats prenatally irradiated on the 12th, 14th, 15 th $(150 \mathrm{R})$, or 17 th day ( 150 or $200 \mathrm{R})$ ? and (ii) Would answers to the foregoing help establish correlations between the development of structural and motor abnormalities? Most 12th-day-irradiated rats showed normal locomotion on difficult paths and nearly normally developing brain and cord structure, but rare CS neurons had bifurcated apical dendrites. Fourteenth and 15 th-day rats had a thin cortex, a large subcortical ectopia, a malformed spinal cord, and a hopping gait. They showed diminished numbers of CS neurons in the cortex with persistent gap CS neurons, and CS neurons in the ectopia that mirrored those in the cortex. Hopping seemed to be generated in the cord, but supraspinal influences require further study. Seventeenth-day rats usually showed disordered locomotor rhythm and inability to adapt it to difficult paths, suggesting impairment of corticostriatal circuits. Some rats also showed inability to place limbs and feet during locomotion on difficult terrain, which resembled that following ablation of areas 3,4 , and 6 . Dorsal cortex, including area 10 , the chief source of corticostriatal projections in the rat, and areas 3, 4, and 6, was most severely malformed. Discrepancies between functional results of ablating area 10 and malforming it with radiation led us to suggest that abnormal thalamocortical and cortical wiring, for which there was evidence, as well as deficits, might be responsible for the abnormal locomotion.

Abbreviations: $\mathrm{HRP}$ - horseradish peroxidase, $\mathrm{CS}$ - corticospinal, $\mathrm{MSC}$ - motor-sensory cortex, H3T-tritiated thymidine.

${ }^{1}$ This research was supported by U.S. Public Health Service grant NS10531. We thank Christos Kanellitsas for calibrating the radiation exposures. 


\section{INTRODUCTION}

The corticospinal or pyramidal tract, that part of the axonal projections of the motor-sensory cortex that extends as far as the medulla and spinal cord, is in the rat one of the largest in proportion to brain size among mammals. Running in the dorsal funiculus of the cord, its greatest development occurs after birth, and it shows a capacity for plasticity of growth after injury to the motor-sensory cortex in infancy. When one motor-sensory cortex (MSC), or that parcel of it that projects to the cord, was ablated in infancy, the corticospinal (CS) neurons in the other MSC formed a compensatory small uncrossed tract, which may possibly have spared animals so treated from a slight impairment of stride that followed the same operation in adults $(11,12)$. Bilateral ablation of the MSC in infancy did not appreciably affect locomotion on flat level surfaces, but profoundly affected skilled locomotion necessary to traverse narrow paths and prevented jumping from one platform to another (12). Adult CS neurons projecting to the spinal cord, identified by retrograde labeling with horseradish peroxidase (HRP), formed two bands separated by a gap, one caudal corresponding to cortical areas 3,4 , and 6 and another rostral in area 10. It was estimated that the axons of about 10,000 neurons in each cortex attained the spinal cord; optimally the HRP method could label one-half to two-thirds of them. CS neurons in the rostral band projected as far as C7 and those in the caudal band formed a mixed population projecting to cervical and lumbar levels (14). In infant rats the gap also contained neurons whose axons attained the cervical cord, but these projections normally disappeared just before the postnatal age of 2 weeks. If the caudal band was injured, the spinal axons of the gap neurons persisted, but their function is unknown (4). Previous work also established that prenatal and early postnatal whole-body irradiation with single exposures of 100 to $300 \mathrm{R}$ yielded a highly reproducible series of malformations, depending on the stage and dose, involving the cortex, striatum, diencephalon, cerebellum, spinal cord, and other structures, in various combinations. Many details of the morphogenesis of the malformations have been worked out $6-9,15,21$ ). Disturbances of gait and other motor behavior were usually observed, but explanations of how the various patterns of altered nervous system structure caused the abnormal functions were elusive, as they so often are in complex developmental disorders $(5,15,18,20,21,26)$. In a very few fetal stages, after severe cellular injury from $150 \mathrm{R}$, there was a remarkable degree of recovery resulting from the regulative capacity of residual primitive cells, and the animals at maturity appeared to be nearly normal as far as they could be studied $(6-9,15)$. Labeling CS neurons with HRP in these irradiated rats in preliminary experiments in search of more 
detailed knowledge of the development of their motor systems showed different patterns of distribution of the neurons in the bands and gap, depending on when the radiation was given $(2,3,13)$.

These experiments raised many questions about the growing central nervous part of the motor system and how its potential for plasticity often failed to adapt to early injury morphologically and functionally. Improved methods for tracing developing nervous pathways and describing movement may help to answer some of the questions. The MSC connects directly or indirectly with almost every part of the central nervous system, from the striatum and diencephalon to the brain stem and cord, through extended projections and collaterals of its axons (12); identification of a major component of it, the CS neurons, may provide insights into the mechanisms that govern its developmental organization. We describe in this paper the postnatal development of CS neurons, and other morphologic characteristics in rats irradiated at selected representative stages of fetal development, give an account of the animals' locomotion, and point out some difficulties in making correlations between morphologic and behavioral changes. In the following paper (17), we describe the nervous systems and movements of rats that were irradiated prenatally on the 14th or 15th day of gestation whose form of locomotion was hopping. A major question here was whether the brain or the cord - or both - were at fault in generating this bizarre gait. In other related experiments we are examining the cellular mechanisms of recovery at early stages after radiation injury in utero, the possible functions of gap CS neurons with injury-induced persistent spinal projections, and the normal and abnormal development of CS and basal ganglia neurons by autoradiographic and other techniques.

We have made preliminary reports of some of these experiments $(2,3$, $13,16)$.

\section{METHODS}

Animals. "Irish" rats (black with white feet) were the F1 cross between albino females of Wistar origin brother-sister mated for about 80 generations and black-selfed nonagouti nales brother-sister mated for 70 generations. In some related experiments referred to, hooded rats brother-sister mated for more than $\mathbf{2 0}$ generations and derived from the Irish were used. Care and housing of the animals were fully accredited by the American Association for Accreditation of Laboratory Animal Care. The experiments conformed to standards in the Guide for the Care and Use of Laboratory Animals, U.S. Department of Health, Education and Welfare Publication 73-23, 1972.

Radiation. Pregnant rats were exposed whole body, in perforated containers made of 3.2-mm-thick transparent lucite sheets, to $150 \mathrm{R}$, and in 
one experiment $200 \mathrm{R}$, with a Westinghouse Coronado X-ray therapy unit. Factors were $250 \mathrm{kV}$ at constant potential, $9 \mathrm{~mA}$, no added filter, $72 \mathrm{~cm}$ from the source of the middle of the animals' bodies, at $67.9 \mathrm{R} / \mathrm{min}$, with continuous monitoring by a Victoreen dosimeter. Doses were measured in cylindrical wax phantoms corresponding in size to, and equivalent to the tissues of, the pregnant rats in the experimental surroundings. In the previous experiments referred to, the factors were identical or very similar.

Stages of Radiation Exposure and Dose. For this study, two rats were irradiated with $150 \mathrm{R}$ for each of days $12,14,15$, or 17 of pregnancies yielding 81 offspring. Many of the basic patterns of radiation-induced malformations in rats and their morphogenesis were established earlier in lines of albino rats of Wistar origin, Irish rats, and sometimes the hooded rats. In brief, they showed that the spectrum of responses to $200 \mathrm{R}$ (at certain stages, $150 \mathrm{R}$ ) included anencephaly in the stage of neural folds and first pairs of somites, late on the 9th to the 10th days; anophthalmia, sometimes with minor cardiopulmonary anomalies, at 6 to 10 somites, and microophthalmia with little else wrong from 10 to 18 somites, during the 10th day; hydrocephalus, microophthalmia, and certain skeletal and visceral abnormalities from 18 to 25 somites during the 11 th day; nearly normal animals from about 25 to 35 somites after $150 \mathrm{R}$ on the 12th day, but encephalocele of the third ventricle with subcutaneous cerebrospinal fluid and skeletal anomalies, lethal at birth, after $200 \mathrm{R}$; a large subcortical ectopia and reduced cortex from 13 days ( 40 pairs of somites) to 15 days, with forefoot abnormalities in those irradiated at 13 or 14 days; the severest deficiency of the cerebral mantle with a scrambled cortex on the 16th or 17th day; and abnormal development of the cortex with distinctive patterns characterizing the period of 18 to 22 days (term), the outer cortex being most affected from 20 to 22 days. A spectrum of cerebellar anomalies especially involving the folia became conspicuous after irradiation on the 18th or 19th day, continuing past the first postnatal week; and because the anlage of the cerebellum, just appearing between the 13th and 15th days, was vulnerable, some general reduction and simplification of folia resulted (21). Retinal anomalies roughly paralleled the later cerebellar malformations, although other earlier eye problems occurred $(7-9,15)$. The prenatal stages of development of our Wistar albino lines had closely paralleled those described in the stereoscopic atlas of Long and Burlingame (25); the Irish rats lagged between one-quarter and one-half day behind the albinos. Stage for stage, some of the basic patterns of malformation were more severe in Irish than albino rats; for example, the rostral part of the mantle after irradiation on the 17 th day, and the cerebellum from the 13 th day to more than a week after birth, were often more deformed in the Irish. The day of insemination was counted as day 1 of gestation. Stages of prenatal 
and postnatal development were established for both the early Wistar and the present Irish rats, in normal reference series of serially sectioned early fetuses and brains of late fetuses, infants, and older animals. Stages were verified in some of the present experiments by removing two embryos by Caesarean section a few hours after irradiation (21). By adjusting the time of irradiation -6 to $12 \mathrm{~h}$ later with the Irish than in the earlier albino experiments - it was possible to continue the same designations by day of radiation throughout the ycars $(7,9,15,21)$.

We selected the 12th, 14th, 15 th, and 17 th prenatal days as representative of the diversity of responses to radiation, and chose $150 \mathrm{R}$ because $200 \mathrm{R}$ on the 12th day led to death at birth and often caused a high perinatal mortality if given from 13 to 15 days. Twelve days is the period of about 25 to 35 pairs of somites, when the forebrain has not yet developed cerebral vesicles, and recovery of the fetus from the initial injury is known to be often of remarkable degree. Thirteen, 14 , and 15 days is when the cerebral vesicles are first developing but with no cortex forming as yet; $150 \mathrm{R}$ results in the subcortical ectopia, a malformed spinal cord, and the hopping gait. [The gait has been attributed variously to the cord anomaly $(15,21)$ and to a hypothetical faulty alternating locomotor mechanism in the brain (26)]. The isocortex was just beginning to appear laterally during the 16th day and had barely reached the vertex of the cerebral vesicle as a single layer of cells by the 17 th. The mechanisms by which the ectopia of 13 to 15 days was formed from a radiation-induced rosetty periventricular proliferative cell population has been described, as has the morphogenesis of the scrambled cortex of 16 and 17 days $(6,8,15)$.

One litter of rats was irradiated with $200 \mathrm{R}$ on the 17 th prenatal day for comparison with the $150-\mathrm{R}$ rats. We also refer to other $200-\mathrm{R}$ experiments where it is useful. For brevity, rats irradiated on the 12th, 14th, etc., days of gestation may be referred to as 12-day, 14-day, etc., rats.

Behavioral Studies. Motor function, especially locomotion on broad and narrow horizontal paths, jumping from one platform to another, and certain reflexes previously described (12), and swimming in 14- and 15-day animals were observed or recorded in slow-motion (64 frames per second) movies. Six to ten walks the length of each path for each animal were usually photographed. A long, slightly inclined mirror behind the paths made it possible to photograph both sides of the animal at once, showing all four limbs. Paths were $150 \mathrm{~cm}$ long marked at $5-$ or $10-\mathrm{cm}$ intervals and usually elevated $36 \mathrm{~cm}$, with cushions below; the broad path was $9 \mathrm{~cm}$ wide, and the most frequently used narrow path was $12 \mathrm{~mm}$ wide. Another narrow path was made of two laterally apposed parallel dowels $6 \mathrm{~mm}$ wide. A longer broad path $13 \mathrm{~cm}$ wide, $2.7 \mathrm{~m}$ long, and curved (for movies) on a $3.1 \mathrm{~m}$ radius was sometimes used. The jumping platforms, elevated $22 \mathrm{~cm}$ 
and cushioned with rubber, were round and $13.5 \mathrm{~cm}$ in diameter for takeoff; comparable landing platforms were rectangular, $10 \mathrm{~cm}$ long by $5 \mathrm{~cm}$. The rats were introduced to these apparatuses when 10 to 14 days old; they gradually learned to walk on the paths and jump increasing distances on the platforms, normal animals showing a transition to adult locomotion on the paths at about 17 days and jumping about $20 \mathrm{~cm}$ by the age of 3 weeks. Reinforcement was the opportunity to explore and be handled $(11,12)$. Tactual placing was tested by touching the lateral aspect of the forefoot to a ledge with the ipsilateral eye shielded by the observer's fingers or a mask. Swimming was observed in mature animals by immersing them in a large tank of water at $38^{\circ} \mathrm{C}$. Some other tests were also used for 14- and 15-day rats that are described in the following paper (17).

Ordinary locomotion on a broad, level, flat surface in the rat has been described as a symmetrical gait characterized by lateral sequence and diagonal couplets $(22,23)$; that is, both right feet moved in sequence, then both left feet, and the movements of the pairs were precisely coupled. Each of the four feet at a given speed contacted the ground for the same time interval. This gait was quickly assumed at about the 17 th postnatal day, within about $24 \mathrm{~h}(11,12)$. To a speed of about $80 \mathrm{~cm} / \mathrm{s}$ the gait was technically classed as a walk [slow, moderate, or fast $(22,23)$ ], rather than a run as it has sometimes been called. Rats can gallop, and this began at about 5 or 6 weeks of age in ordinary laboratory circumstances (12).

A method for recording locomotion in graphic form was to plot gait diagrams from successive movie frames to show the stance time (when the foot is on the ground) and the swing time (when the foot is off the ground) of each of the four limbs in relation to one another. Stance time plus swing time of a complete locomotor cycle of a limb was the stride interval. The length of stride was the distance between two successive footfalls of the same limb. A number of variables were defined based on the relationships recorded in the gait diagram (22), for example, the percentage of the stride that the footfall of a forefoot followed the ipsilateral hind foot, when the durations of footfalls for all limbs were equal. Another relationship, when hind- and forefoot contacts were unequal in duration, concerned the instants of maximum support by the respective legs, that is, the midpoint in time of the respective footfalls. A variable in this relationship was the percentage of the stride interval that forelimb midtime followed the midtime of the ipsilateral hind limb. Calculating these variables did not aid us, for the most part, in analyzing the gait of the irradiated rats, but gait diagrams graphically contrasted the hopping 14- and 15-day rats with those irradiated on other days and with normal animals (17).

We extended the use of gait diagrams to locomotion on a narrow path. A revealing measure suggested by the appearance of the gait of 17-day rats in 
movies was the lengths of several strides of the two hind limbs recorded in alternate succession, that is, left, right, left, and so on. From the movies, the successive positions of the center of the foot were measured on the tracks, which were marked every 5 or $10 \mathrm{~cm}$, and the length of the stride was estimated to the nearest centimeter. Speed was estimated from the number of movie frames (at 64 frames per second) for the animal to traverse a given distance. As it moved, the rat was held fairly well centered in the camera view-finder frame adjusted to include $30 \mathrm{~cm}$ of the track. Small animals change their speed more than large animals (22), but substantial trains of steps at uniform speed could usually be readily obtained (23), except from those animals whose gait was very abnormal. The standard deviation of the mean of the sample of consecutive left and right hind-limb strides gave an indication of their variability. A single stride very different from the others in a train of eight could increase the standard deviation; thus several trains were examined. Normal rats virtually never showed this kind of single, large deviation in stride. A representative example of a train of strides, their mean $\pm \mathrm{SD}$, and speed of a normal rat at a moderate, almost fast walk on a narrow track, starting with the left hind foot (LH), illustrates how these data are presented: normal LH 15-13-12-14-14-13-13-14-14 cm, $13.5 \pm 0.9 \mathrm{~cm}, 57 \mathrm{~cm} / \mathrm{s}$.

Anatomy and Histology. The animals were studied by gross examination, regular histologic methods, and retrograde transport of HRP to label CS neurons. To understand better the genesis of certain malformations, appropriate material was used from a continuing study with tritiated thymidine (H3T) histologic autoradiography to trace the mitotic cycles and proliferation of fetal neural cells, their migrations and differentiation, and the effects of radiation on them.

For gross anatomy and regular histologic studies, mature animals were killed and autopsied. Brains and blocks of spinal cords were usually fixed in Bouin's solution, sometimes in 10\% Formalin in saline, paraffin embedded, and sectioned serially at $8 \mu \mathrm{m}$. Representative sections, sometimes alternate or all sections, were stained with luxol fast blue and cresyl violet or hematoxylin. Some tissues were prepared by frozen sections for Cajal pyridine silver stain, and others for Golgi stain. A sagittal half of each brain was serially sectioned, usually sagittally, and the other sometimes frontally. Small blocks of spinal cord of upper, middle, and lower cervical, midthoracic, and upper and lower lumbar levels were studied in transverse sections by these methods. In comparing brains and cords of irradiated and normal rats, we took into consideration the different degrees of shrinkage induced in gross brains, paraffin-embedded sections, and frozen sections, as measured previously (14). Two to four adult 12-, 14-, 15-, or 17-day and normal rats were represented. Some fetuses were removed surgically to 
confirm the stage at which radiation was given and to observe the initial injury $(15,21)$.

Experiments to identify CS neurons with HRP were carried out, as recently described in detail $(4,14)$, in rats 7 or 10 days old and adults. Briefly, HRP crystals were introduced into the wound of a surgical transection of the dorsal part of the upper cervical (between $\mathrm{C} 1$ and $\mathrm{C} 3$ ) or lumbar (L1) spinal cord, including the CS tracts, of the anesthetized rat. Fixation was by intracardiac perfusion with buffered aldehydes with the animal anesthetized with ether. Frozen serial sagittal or frontal sections, 40 $\mu \mathrm{m}$ thick, of the brains were collected in five jars and sections of two or more jars were reacted with diaminobenzidine for dark-field examination or with dianisidine and occasionally other benzidine derivatives counterstained red with Mayer's hematoxylin or Darrow red for light-field examination. For each day of irradiation with $150 \mathrm{R}, 12,14,15$, or 17, usually two, sometimes one or three, infant animals were examined at 7 or 10 days of age or both, and two to six adults were used. Two or more normal animals were used to match each age. For comparison, two rats that received $200 \mathrm{R}$ on the 17 th prenatal day were also treated with HRP. The HRP was introduced between $\mathrm{C} 1$ and $\mathrm{C} 3$ in all animals. One additional adult rat irradiated with $150 \mathrm{R}$ on the 12th, 14th, or 17 th day, and several normals, received HRP at $L 1$.

Histologic autoradiographic studies were done by administering H3T to rats pregnant on one of the gestational days 11 to 22 and surgically removing two or three fetuses from the same litter at chosen intervals, or allowing some pups to be born for postnatal and adult samples. This provided sequences of stages of development for each gestational day in which the stepwise progress of populations of cells that took up H3T could be followed through their mitotic cycles, migrations, and differentiation to their destinations. Combined with radiation at certain periods after H3T was given, vulnerable periods during the mitotic cycles, migration, and differentiation could be inferred. The methods and some results have been published $(9,10,15,19)$ as background for the present continuing series of experiments.

\section{RESULTS}

\section{Behavioral Studies}

Irradiation on the 12th Day. Ten of 18 rats irradiated with $150 \mathrm{R}$ on the 12th prenatal day were observed as mature animals. Movies of the locomotion of six were analyzed. With one exception, a runt animal with certain defects described later, the rats traversed the broad and narrow 
paths as well as the normal controls, as seen in the movies or by direct observation. There was no abnormal positioning of the limbs and feet. Gait diagrams of some irradiated rats on narrow paths showed a rhythm indistinguishable from that of the normal controls (17). Measurements of the hind-limb strides showed nearly uniform lengths of strides of both limbs, with no greater variability than normals (Table 1). Seven rats jumped variable distances to $30 \mathrm{~cm}$ on the platforms in various directions with the same ease and accuracy as normals. Like normals, they took off equally with the hind feet and held the forelimbs in partial flexion during the jump, extending the forefeet just as they landed, forefeet together. They tactually placed normally. The three rats that were not tested in jumping had small eyes; one was the runt rat, and the other two were nearly normal in other respects. The runt animal walked with small steps, but in an otherwise normal manner.

Irradiation on the 14th or 15th Day. Thirty-one rats irradiated with $150 \mathrm{R}$ on the 14th or 15th prenatal day were observed; movies of 15 of 24 mature rats were studied. All 14- and 15-day rats showed a hopping gait (Table 1), evident at about the time of birth (17). The paired hind- and forelimbs moved synchronously instead of in the normal alternating mode. For the first 2 days of life, when limb movements are normally apt to appear random, the "hopping" could be seen in hind- and forelimb pairs when the animals were crawling, especially if the hind or fore part of the body was

\section{TABLE 1}

Consecutive Strides of the Left and Right Hind Limbs in Normal and Prenatally Irradiated Rats on a Narrow Path (Rectangular) ${ }^{a}$

\begin{tabular}{llrr}
\hline \multicolumn{1}{c}{ Irradiation } & \multicolumn{1}{c}{$\begin{array}{c}\text { Gait } \\
(\mathrm{cm})\end{array}$} & $\begin{array}{c}M \pm S D \\
(\mathrm{~cm})\end{array}$ & $\begin{array}{r}\text { Speed } \\
(\mathrm{cm} / \mathrm{s})\end{array}$ \\
\hline (A) Normal & LH 13-14-14-14-16-14-13-15 & $14.1 \pm 1.0$ & 77 \\
(B) Normal & LH 15-13-12-14-14-13-13-14-14 & $13.5 \pm 0.9$ & 57 \\
(C) 12-day 150 R & LH 13-12-13-12-14-13-12-13-11-13-13-12 & $12.5 \pm 0.8$ & 52 \\
(D) 12-day 150 R & RH 13-13-14-13-12-13-12-13-14-12-14-14 & $13.1 \pm 0.8$ & 53 \\
(E) 17-day 150 R & LH 13-13-13-13-12-16-13-11-13-15-11-11-15 & $13.0 \pm 1.6$ & 51 \\
(F) 17-day 150 R & RH 11-11-13-13-13-13-13-13-13-12-12-11-11 & $12.2 \pm 0.9$ & 43 \\
(G) 17-day 200 R & RH 8- 9-11- 7-11-11-11 & $9.7 \pm 1.7$ & 38 \\
(H) Normal & RH 17-16-20-20-20-20-18-19-16 & $18.7 \pm 1.6$ & 94 \\
(I) 14-day 150 R & LH 7- 7- 7- 7-7-7-7-7-8- 8- 8- 8 & $7.4 \pm 0.5$ & 45 \\
\hline
\end{tabular}

a A-G-LH, RH (left or right hind foot) indicates which foot began the sequence. H-The flawless run (running is greater than $80 \mathrm{~cm} / \mathrm{s}$ ) of a normal rat illustrates the effect of acceleration and deceleration on a broad path. The mean and standard deviation of the six strides from 20 to $19 \mathrm{~cm}$ were $19.5 \pm 0.8 \mathrm{~cm}$. I-The hind- and forelimb hopping of this 14-day rat is not revealed by this kind of measure. 
lifted by the observer. Thereafter it became more obvious. Of the 24 animals observed to maturity, about half switched more or less completely to an alternating gait of the forelimbs during about the third and fourth weeks of age, retaining the hopping in the hind limbs. Rarely, a few rats took a single alternating step with the hind limb, then resumed hopping. One animal switched to a normal alternating gait of all four limbs most of the time, only rarely hopping briefly. A few animals were severely affected by their gait. All animals could swim, the "gait" being that used by the particular animal on a flat terrestrial surface. Twenty-two rats were tested in jumping. All could jump 20 to $25 \mathrm{~cm}$ from one platform to another in the manner of normal animals, except the few severely affected, which jumped shorter distances. On narrow paths, the hopping animals hopped, those severely affected being very clumsy, their hind limbs often slipping off. We give a detailed account of these animals, including other tests made, in the following paper (17).

Irradiation on the 17th day. The locomotion of eight rats irradiated with $150 \mathrm{R}$ and 12 irradiated with $200 \mathrm{R}$ was observed and examined with movies. Gait on the different tracks varied among these animals, ranging from nearly indistinguishable from normal in two 150-R rats through moderately abnormal in five $150-\mathrm{R}$ rats to markedly abnormal in most of the 200 R-rats. The abnormal characteristics of the animals" locomotion can best be described in words supported by numeric measures of the hind-limb strides and other gait components.

The locomotion of a normal rat from about the 17th day of age was precisely rhythmic, consistent over substantial stretches, and adapted to the terrain. The positions of every part of the body from nose to tail were integrated with utmost smoothness into the action. The two nearly normal rats differed from normals only in being a little less smooth and losing their footing more often, characteristics we could not quantify or describe precisely. All other 17-day rats, when seen in slow motion, showed in varying degrees a disturbed rhythm and inconsistent maintenance of one or more components of the gait over substantial stretches. Locomotion as a whole lacked the smooth flowing appearance of the normal, and in those most affected it was ill-adapted to the terrain, especially the narrow path. The disturbed rhythm could often be measured by the variability of the length of stride in the two hind limbs of several animals (Table 1) and by describing other deviations in gait in others. In all 17-day rats some strides, and in a few animals most of the strides, were too long: The limb was swung forward as far as it could reach, and at the end of stance it was stretched out as far behind as possible. This was sometimes accompanied by an exaggerated lateral flexion of the lumbar spine to bring the swinging hip forward. Not infrequently the hip in swing phase was lifted 
unusually high, and at times the corresponding hind foot was swung in an arc outward as it was carried forward. These abnormalities were seen on both the broad flat track and the narrow paths. In a gait diagram of one 200$R$ rat on the rectangular path, swing time as well as stance time was especially variable (17).

The disturbed gait resulted in poor adaptation of the rats' locomotion to difficult terrain represented by the rectangular narrow path, and all 17-day rats lost their footing more frequently than normals. One 150-R rat could scarcely negotiate the narrow paths, and another was nearly as severely affected. Three others were quite clumsy, but produced some sustained walks with variable strides. Although the sixth lost its footing frequently, it managed several sustained walks with no more variability of measured strides than normal, but on the flat broad path showed abnormal variability and extended stride. Of the 200-R rats, seven had so much difficulty on the narrow paths that no sustained walks long enough for movie analysis occurred. Three others could occasionally keep their feet for sustained walks with variable stride, and two managed some substantial walks. Many animals attempted to gallop briefly when they could make no headway after repeatedly losing their footing.

Another characteristic appeared in two of the 200-R rats: They occasionally let their hind or forelimbs dangle briefly when they had slipped off the narrow path or when they walked along the edge of the broad path. This was a characteristic of rats whose posterior medial quadrant of the motor cortex was removed unilaterally in infancy or adulthood (12). This region included areas 3,4 , and 6, the principal source of CS neurons projecting to the spinal cord and posterior column nuclei, and its removal produced a contralateral loss of tactual placing in the immobile animal and what we called impaired locomotor placing or dangling in the contralateral limb when the animal was in motion along the edge of a broad track or on a narrow path.

The 150-R rats jumped from one platform to another as well as normals. The 200-R rats were not tested for jumping. The 150- and 200-R rats tactually placed when, as the animals were held with vision shielded, the lateral part of the forefoot was touched to a ledge. The two 200-R rats mentioned previously that dangled their limbs resembled, in this respect, rats whose motor-sensory cortex was bilaterally ablated at birth. Such ablated rats tactually placed when held, but exhibited impaired locomotor placing when they walked.

The other rats that had a generally difficult time on the narrow track retrieved their limbs promptly when they lost their footing and replaced their feet well, only to lose their footing again and again after they resumed the rhythmic movements of locomotion. 


\section{Anatomic and Histologic Studies}

We describe certain features of malformations of the central nervous system and other structures of the mature rats exposed prenatally to $150 \mathrm{R}$ on the 12th, 14th, 15th, or 17th day or $200 \mathrm{R}$ on the 17th day including the identification and location of CS neurons with HRP in infancy and maturity.

Irradiation on the 12th Day. All 22 animals in the two litters appeared superficially normal except as follows. In one litter, one showed hydrocephalus and died at birth; it was assumed to have been younger than its mates at the time of irradiation. In the other litter, one was a runt with misaligned jaws which allowed the teeth to grow, requiring frequent clipping. Its eyes were small. Found dead, its brain at a year of age was not hydrocephalic, appearing grossly unremarkable. Two other animals appeared normal but had small eyes. Another animal was removed as a fetus $24 \mathrm{~h}$ after radiation, showed the expected changes due to the exposure, and will be considered in another study (D'Amato and Hicks,
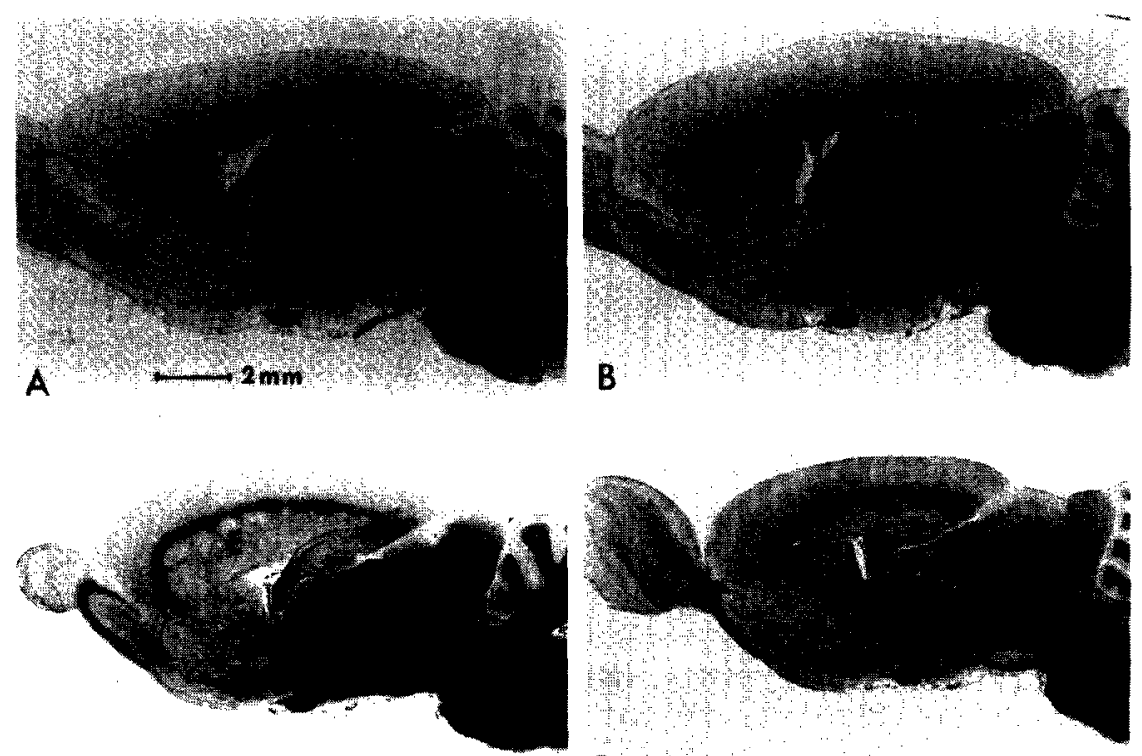

C

D

Fig. 1. Sagittal sections of the brains of prenatally irradiated adult rats compared with normal. A-normal. B-150 R, 12th day. C-150 R, 14th day. D-150 R, 17th day. The 12-day rat (B) is virtually indistinguishable from normal, despite severe radiation damage as an embryo. The 14-day rat $(C)$ has a large subcortical ectopia (E), thin cortex, small olfactory bulb, and slightly simplified cerebellum. The 17-day rat (D) has a much reduced pallium. Luxol fast blue and cresyl violet. 
in preparation) of recovery in fetuses irradiated at 12 days. The animals were studied grossly and by the various histologic methods described (Figs. 1, 2). The brains of animals prepared in paraffin sections were indistinguishable from normal. As normal as these animals appeared to be, we were able to find four, possibly five, kinds of abnormalities. (i) Some rats appeared to have slightly shorter necks than normal. (ii) The spinal cord of some animals, but not others, was a little smaller in diameter in the cervical region than normal. (iii) One animal had very slightly enlarged lateral ventricles and a reduced brain length but otherwise appeared unremarkable. (iv) CS neurons occasionally showed bifurcated apical dendrites in HRP preparations. (v) Qualitatively, 12-day rats appeared to have fewer CS neurons than normal as shown by HRP labeling.

The cervical intumescence of the spinal cord was about 4 to $5 \mathrm{~mm}$ in greatest width in irradiated animals, whereas in normals it was about 4.5 to $5 \mathrm{~mm}$. Other levels of the cord appeared unremarkable. Spinal ganglia did not appear different from normals, but measurements of these structures were difficult to quantitate. Histologically, as described in detail in the following paper (17), the normal-size cords appeared normal and the
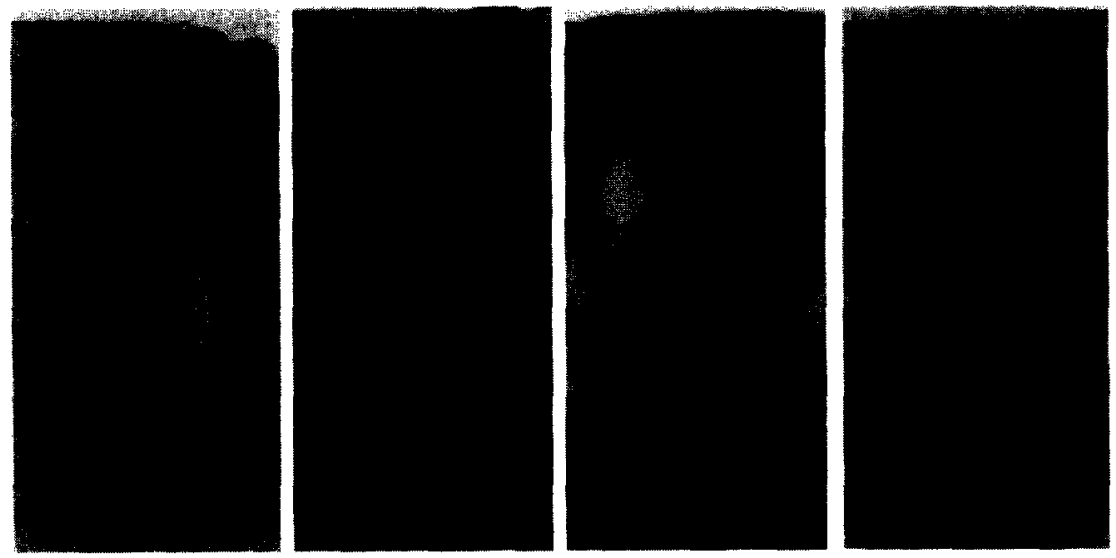

Fig. 2. Sagittal sections showing the midportion of the caudal band of the dorsal cortex of adult rats. They show corticospinal (CS) neurons labeled with horseradish peroxidase (HRP) from the cervical cord in A-C and the lumbar cord in D. A-normal. There were $105 \mathrm{CS}$ neurons in the band in the microscopic section represented. There are especially heavy deposits of HRP reaction product. B-150 R on the 12 th prenatal day. There were $83 \mathrm{CS}$ neurons in the band in the microscopic section, with less reaction product than in $A$. $\mathrm{C}-150 \mathrm{R}$ on the 14 th prenatal day. CS neurons are present in the cortex above and in the ectopia (E) below. D-150 R on the 17th prenatal day. A few, mostly lightly labeled, CS neurons are present in the middle of a faintly visible disordered cortex. Dianisidine and hematoxylin. 

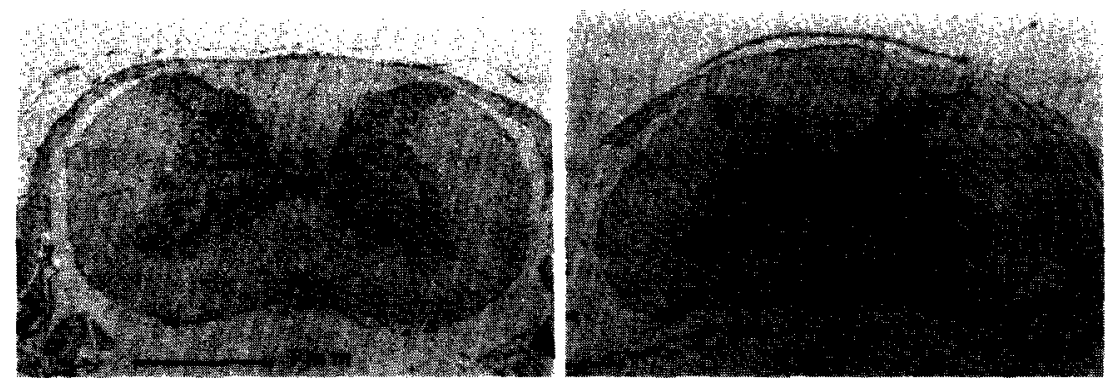

FIG. 3. Cross sections of the cervical spinal cords, between $\mathrm{C} 4$ and $\mathrm{C} 5$, of an adult rat irradiated with $150 \mathrm{R}$ on the 14 th prenatal day, compared with a normal, left. The gray commissural region is thickened and the posterior horns, $\mathrm{PH}$, are reduced in the irradiated animal. The corticospinal (CS) tracts are faintly visible. Cresyl violet.

smaller cords in irradiated animals appeared normal except for a general proportional diminution of gray matter. The CS tracts, composed of small, fairly uniform, closely packed fibers, were distinctive in the base of the posterior columns in any preparations stained for myelin sheaths or axis cylinders, and faintly visible in cell stains (Fig. 3). They appeared nearly normal.

In three 7-day old and two 10-day old infants given HRP in the cervical cord, the distribution of CS neurons was the same as in normal controls, the gap region containing labeled CS neurons (Fig. 4). In mature animals, the CS neurons labeled from the cervical cord were virtually absent or quite sparse in the gap and present in the bands as in normals. Figure 2 includes examples from the middle of the caudal bands in a normal and a 12-day rat. There were 105 labeled $C S$ neurons in the caudal band in the microscopic section represented in the illustration of the normal (A) and 83 in that of the 12-day rat. The lighter labeling and seemingly smaller neurons in the 12-day rat (B) are due to a lighter deposit of HRP reaction product than in A. The apparently fewer labeled cells in all 12-day rats, as represented in this animal, may or may not be significant; our method of labeling gave semiquantitative results and the variance among normals was large (14). Total CS cell counts were not made on these 12-day rats. The one mature animal labeled from $\mathrm{L} 1$ resembled normals in that neurons were distributed through the caudal band but not the rostral. Bifurcation of apical dendrites of CS neurons (labeled with HRP) was occasionally seen; it was never seen in a large number of normal adult and infant rats.

Most of the 12-day brains were serially frozen-sectioned for HRP experiments by the methods described, providing less opportunity to make detailed comparisons of different parts of the brain than in paraffin sections. The overall size of the brain, the greatest length of the thalamus 

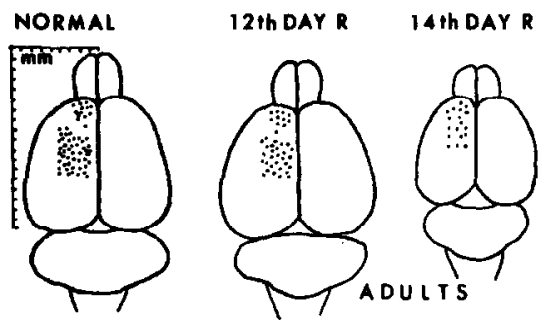

17th DAY R
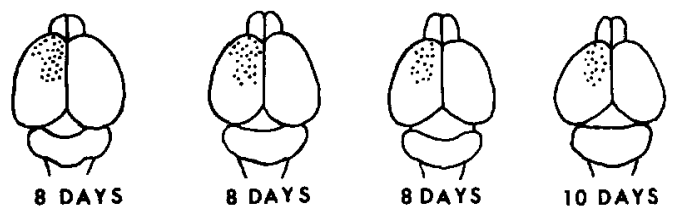

Fig. 4. Diagram to show with dots the distribution of corticospinal neurons in the dorsal cortex of 8- or 10-day-old infant and adult rats prenatally irradiated with $150 \mathrm{R}$ compared with normal. Rostral and caudal bands separated by a gap are shown in the adult normal and 12-day irradiated rats; all others show cells in the gap as well as band regions. CS neurons in the ectopia of 14-day irradiated rats lay precisely beneath those in the cortex in this perspective. Freely reconstructed from microscopic sections.

and striatum, and the structure of the cortex appeared similar to those features in the normal, except in the one animal with slightly enlarged lateral ventricles whose thalamus and striatum were shorter than the normal range.

Irradiation on the 14th or 15th Day. These animals were grossly sometimes about the same size as normals, but more often were somewhat smaller. Fourteen-day rats, like 13 -day rats previously described $(5,7,21)$, had deformed toes of the forefeet, substantially reduced cerebral hemispheres with a large subcortical ectopia, and malformation and deficiencies of the posterior horns of the spinal cord. Fifteen-day rats were basically similar, but lacked the foot deformities. The ectopia, beneath the overlying dorsal cortex, extended the dorsal length of the lateral ventricle, whose roof it formed. A band of fibers corresponding to the centrum semiovale, but running in all directions in the form of bundles, and well seen in sagittal sections (Fig. 1), separated the ectopia for the most part from the overlying cortex. There was little or no corpus callosum formed, as was found in previous studies of 13-, 14-, and 15-day rats (21).

Aberrant bundles of fibers, of the type seen in 16- and 17-day rats (21), traversed the cortex of 15-day rats more than in 14-day rats, but appeared in the ectopias of both. The fibers of the thalamic radiation and corticofugal fibers that formed bundles in the striatum coursed around the ectopia 
posteriorly, laterally, and frontally. The striatum was somewhat depressed along its dorsal border by the ectopia, but its volume was little reduced from normal as estimated from measuring its greatest length and breadth. The cerebellum showed some simplification of subdivisions of the lobules and was sometimes a little narrower and usually shorter longitudinally than normal. The shortening of the anterior-posterior dimension of the vermis was more noticeable in 15-day rats. The spinal cord was deformed, there being an overall deficiency of the dorsal gray columns involving Rexed's laminas I to VI and possibly the dorsal part of VII. Often there was a partial fusion of the medial basal parts of the dorsal horns, making the central gray commissure very thick (Fig. 3). The CS tracts in the spinal cord were variably but substantially reduced. This is just discernible in the cresyl violet (Nissl) stain in Fig. 3. We described previously the morphogenesis of the malformed cord (7) and of the subcortical ectopia, from the rosetty periventricular proliferative cells that followed cell destruction caused by the radiation $(6,8,15)$, and we give a detailed account of the spinal cord in the following paper (17).

HRP labeling from the cervical cord showed that CS neurons developed in both the normally situated cortex and the ectopia (Fig. 2). The cells were disposed in a generally normal manner in the middle of the cortex, being components of a layer $\mathrm{V}$, but they were distributed not only in the caudal and rostral bands but also in the gap in both mature rats and 7- and 10-day-old infants (Fig. 4). The number compared with normals was greatly reduced in all animals and was undoubtedly significant. Many fewer CS neurons were scattered at all depths of the ectopia, oriented in every direction and appearing pyramidal, irregularly stellate, or spindle shape. In contrast, their longitudinal and lateral position within the domains of the ectopia conformed precisely to the overlying CS neurons in the cortex: They lay exactly and only beneath the bands and gap. The adult animal labeled from $\mathrm{L} 1$ showed a few CS neurons in the cortex and ectopia corresponding to the caudal band.

Irradiation on the 17th Day. Rats irradiated on the 17 th day with $150 \mathrm{R}$ were usually somewhat smaller than normal as they matured. The animals' skulls dorsally were proportionately flatter and smaller than normal. The cerebral hemispheres were small and the striatum, base of the brain, and diencephalon were very little affected, when the brains were sectioned grossly, and no significant abnormality of the cerebellum and brain stem was evident. Microscopically the vertex of the mantle, that is, the dorsomedial, dorsal, and dorsolateral cortex, which we will call dorsal hereafter, was the most affected part of the brain (Fig. 1). The cytoarchitecture of this part of the cortex was disordered, and the thickness of the cortex reduced. The laminar arrangement of cortical neurons was 
considerably obscured, with the large pyramidal neurons of layer $\mathrm{V}$, for example, distributed over a broader zone than normal and sometimes displaced into the more superficial and deeper layers. Polarity of neurons was commonly disturbed, deviation from the vertical in respect to the cortical surface sometimes ranging to a horizontal position. Inverted neurons were rare. In a Golgi preparation in one animal, the semblance of normal lamination was more clear-cut because cell types were better defined. Ectopic neurons in layer I, sometimes in groups, were not infrequent. Intermixed with the disordered neurons were aberrant bundles of fibers sometimes projecting as far as the cortical surface, and extending in all directions. We showed earlier that many bundles were the result of disordered development of thalamocortical afferent fibers (21).

Two hundred-roentgen rats showed a greater reduction in the thickness of the cortex than 150-R rats; the disorder of the arrangement of the cells in both 150 - and $200-\mathrm{R}$ rats could fairly be described as scrambled, reflecting the manner in which they were produced in fetal life. The clustering, displacement of neurons, and disturbed polarity were more marked in 200-R rats. Neurons were not infrequently upside down. In 200-R rats it was often especially evident that the rostral dorsal part of the MSC was the most severely affected. In a few animals this part of the mantle was extremely thin.

There was a minute subcortical ectopia rostrally in 150- and 200-R rats, corresponding to the large one in 13- to 15-day rats. The lateralmost isocortex and cortex below the rhinal fissure, being more mature at the time of irradiation, were much less affected than the dorsal cortex. The brain stem and cerebellum were essentially normal with respect to the overall size of the brain. The dorsalmost parts of the posterior horns of the cord were slightly reduced (17). The CS tracts in the spinal cord were reduced substantially as seen in cross section.

CS neurons were formed in the dorsal isocortex corresponding to the domains of the rostral and caudal bands and in the gap, in both infant and mature rats, when labeling was from the cervical cord. They were sparser than normal in all animals. A fair number of these labeled CS neurons had bifurcated apical dendrites; correspondingly, in Golgi preparations scattered pyramidal neurons throughout much of the dorsal and dorsolateral isocortex had similar apical dendrites. Although the CS neurons tended to be in a "layer V," occasionally they were found as high as layer II and oriented in various directions. In the two $200-\mathrm{R}$ rats labeled with HRP, whose cortices were quite scrambled, labeled neurons were sometimes scattered as far as what would correspond to layer I or deep layer VI, and they were occasionally inverted. In the $150-\mathrm{R}$ adult animal labeled from $\mathrm{L} 1$, the disposition of the CS neurons corresponded to layer $\mathrm{V}$ 
of the caudal band and were sparse (Fig. 2). No labeled neurons were seen in the rostrally situated tiny ectopias.

The volumes of the striatum and diencephalon were estimated to be relatively little reduced from normal judging from the length, height, and breadth of these spheroid structures in either the 150- or the 200-R group. A very brief account of $\mathrm{H} 3 \mathrm{~T}$ autoradiographic and other studies (Hicks and D'Amato, unpublished observations) shows in part how this outcome resulted. Production of the large neurons for the caudate-putamen and the globus pallidus-entopedunuclar nucleus began during the 12th prenatal day and subsided during the 17 th, few small neurons being generated during these several days. On the 18th and 19th days there was a resurgence of proliferative activity and vast numbers of small neurons were produced, and again on the 20th and 21 st days large numbers were formed. These various cells, of course, had different destinations and different transit times to reach them. Most of the thalamic components were generated in the 12th- to 16th-day period, as were those of the substantia nigra, zona incerta, subthalamic nucleus, and nucleus accumbens, the latter continuing to receive cells generated as late as the 20th day. What was especially interesting was that the proliferative cell population programmed to produce caudate-putamen neurons after the 17th day was able to recuperate from the 17 th-day irradiation with 150 or $200 \mathrm{R}$ well enough to provide subsequently most of the small striatal neurons. At the same time numerous members of another proliferative population concerned with making a cortex, as well as many of the still primitive cells that originated from the same source a day or two earlier, and now en route to form the deepest layers of the dorsal cortex, were being destroyed. The resulting disruption of the early laying down of the cortex, as we described previously $(8,15)$, led to a scrambled cortex.

\section{DISCUSSION}

\section{CS Neurons}

The CS neurons projecting to the cord developed in the cortex of 12-day rats in a cytoarchitecturally normal manner, the gap neurons giving up their cord projections as the animals matured. The gap neurons persisted in the 14-, 15-, and 17-day rats; these rats certainly had fewer CS neurons than normal. The relative lack of CS neurons in the caudal bands may have been a stimulus to the infant gap CS neurons to keep their cord projections, as we postulated might be the case in rats whose gap neurons kept their cord projections after partial ablation of the caudal band in infancy (4).

Bifurcation of apical dendrites occurred in some CS neurons in 12-day and 17-day rats, and we previously observed bifurcation and other 
abnormalities of apical dendrites of many layers II and III neurons in the dorsal isocortex in rats irradiated on the 20th or 21 st prenatal day $(9,15,18)$. CS neurons in the ectopias of 14- and 15-day rats were sometimes clearly abnormally shaped, such as long spindle forms not attributable to the plane of section. CS neurons were chiefly produced on the 17 th prenatal day, migrating to their destinations in dorsal cortical layer $\mathrm{V}$ during the succeeding 2 to 4 days (10). Thus, in the 12-, 14-, and 15-day rats only the remote ancestors of CS neurons would be exposed to the radiation; on the 17 th day the CS neurons, and on the 20th and 21st days, layers II and III neurons, were just being produced and therefore were in their most primitive stage at the time of irradiation. It is unlikely that irradiation of remote ancestors or of neurons in their most primitive stage would have such a common later effect on the shape of the cell. In the 14-, 15-, 17-, 20-, and 21-day rats, the neurons of interest (ectopic neurons in the 14- and 15-day rats) found themselves in anatomically abnormal evironments at the ends of their migrations, and this may have influenced the shaping of the cells. Thalamocortical afferent axons normally developed pari passu with the isocortical neurons and influenced cortical architecture and nerve cell differentiation $(12,21)$; disturbance of this interrelation may have affected dendrite development. At present we can suggest no cause for the unusual dendrites in the 12-day rats.

The ectopic CS neurons in the 14- and 15-day rats were remarkable in that (i) they were able to differentiate as such, (ii) their distribution in the ectopias corresponded to their arrangement in the overlying cortex, and (iii) their axons found their way to the spinal cord. The ectopias, as we showed, were derived from primitive proliferative cell populations which were disrupted by losses from the radiation, and whose residual cells formed multiple rosette centers of proliferation from which the cortex and the subcortical ectopia were built. One way to look at the outcome in these rats is that a program was apparently already set in motion at this early stage to build a pallium with its parcellated cortex, and the rosetty remains of the proliferative cell population still retained enough information to build a caricature of it including a cortex and an ectopic mirror of it. The labeled CS neurons provided evidence of the degree of parcellation that was possible.

\section{Locomotion}

The 12-day rats did remarkably well with respect to locomotor aspects of movement, attesting to the degree of recovery they made from the initial radiation damage. Three rats, as we noted, were not tested on jumping. We defer discussion of the 14- and 15-day rats to the following paper (17) but, in 
brief, we conclude from anatomic considerations, physiologic responses after spinal cord transections, and other behavior that the malformed cord was the chief factor in causing the hopping locomotion, but that supraspinal centers including the cortex adapted to the abnormal spinal locomotor generator and made functional use of it (17).

The principal abnormality in the 17-day rats irradiated with 150 or $200 \mathrm{R}$ was a deficient mantle with a scrambled isocortex, most marked in the dorsal parts of the MSC, especially rostrally. A correlation here between altered nervous system structure and function might stop with the statement that the malformed motor-sensory cortex accounted for the faulty locomotion, but we attempt to carry it further, proposing that a principal fault in the 17-day rats was impairment of cortical-striatalthalamic-cortical loop circuits, believed to be essential for the programming and control of movement $(1,23,27)$. The animals' gait suggests to us that this part of the motor system might be out of order, and because the striatum and thalamus seemed to be little affected, the cortical links in the circuits would have to be at fault. A major characteristic of the gait of these rats was an abnormal rhythm of the cyclic movements of the limbs and an inability to change it to adapt to the exigencies of difficult terrain such as walking on a narrow path. The inadaptibility is somewhat like that of a toy animal with an inflexible gait walking on a track; it can negotiate the track until the limbs and feet are out of line, then lose its footing because it cannot change. The rats (with two exceptions) were generally able to retrieve their feet quickly and place them appropriately on the track after losing their footing, and resume walking, only to falter again sooner or later. The two exceptions were rats that had, in addition, impaired locomotor placing (12): When they lost their footing on the narrow path, or the edge of a broad path, they had difficulty retrieving and replacing the feet, sometimes letting one or more of the limbs dangle. Impaired locomotor placing was characteristic in earlier experiments on rats in which the anterior medial quadrant of the MSC was unilaterally or bilaterally ablated in infancy or adulthood, this region containing the caudal band with the majority of CS neurons that project to the spinal cord and posterior column nuclei. The dorsal cortex, which includes both the anterior-medial and the posterior-medial quadrants of the MSC, was the most deficient and malformed part of the brain in 17-day rats, and the anterior-medial quadrant was more severely affected than the posterior. In some $200-\mathrm{R}$ rats this rostral part of the dorsal mantle was extremely thin. In the rat, the major locus of corticostriate projections is in the anteriormedial MSC quadrant, which includes much of area 10 and the rostral caudal band of CS neurons whose axons do not extend as far as the lumbar region. In contrast, in normal rats there are relatively few projections from 
the posterior-medial quadrant to the striatum. Assuming that we are correct in ascribing the disordered rhythm and adaptibility of locomotor movement to impaired striatal mechanisms, then a case might be made for correlating the severe malformation of the anterior-medial quadrant with the functional disorder. In some animals, the malformation and deficit of the posterior-medial quadrant, including its CS projections, appeared to have impaired locomotor placing, too. However, in some earlier experiments in which we ablated the anterior-medial quadrant unilaterally or bilaterally, rats showed essentially no abnormality of locomotion (11, 12). When we ablated both the anterior-medial and the posterior-medial quadrants, the result was predominantly impaired locomotor placing. The only resemblance of these animals' gait to that of the 17-day rats with respect to locomotor rhythm was that they very occasionally allowed a hind limb to extend far caudally, a posture virtually never assumed by normal rats.

How might the functions of a malformed cortex resemble or differ from those that result from removal of the corresponding normal cortex? If a malformative process is severe enough to be tantamount to ablation, the effects should be similar. At the other extreme, the malformed cortex performs some functions fairly well; Falk showed in our earlier experiments that 200-R, 17-day rats had significant ability to discriminate visually some patterns of the Klüver-Lashley series, and among different concentrations of quinine water, both functions regarded in the experimental circumstances as being cortically dependent $(15,18)$. Those authors also found that 150-R, 17-day rats could also jump varying distances and directions on the platforms under visual guidance. There was a visible disorder of wiring in the 17-day cortices, much of it disordered thalamocortical afferent pathways (21). How much the disordered cortex might have been able to simulate normal function, or interfere with normal function, or create abnormal function of its own is not known. The latter kind of abnormal function might account for the differences between rats with an ablated anterior-medial MSC and a malformed one.

\section{REFERENCES}

1. Carter, D. A., and H. C. Fibiger. 1978. The projections of the entopeduncular nucleus and globus pallidus in rat as demonstrated by autoradiography and horseradish peroxidase histochemistry. J. Comp. Neurol. 177: 113-124.

2. D'AMATo, C. J., AND S. P. Hicks. 1978. Radiation effects on developing corticospinal neurons. Abstracts of VIIIth International Congress of Neuropathology, Washington, D.C., No. 74, p. 605.

3. D'Amato, C. J., AND S. P. Hicks. 1978. Effects of radiation at various prenatal stages on developing rat cortex and corticospinal systems. Soc. Neurosci. Abstr. 4: 74. 
4. D’Amato, C. J., AND S. P. Hicks. 1978. Normal development and posttraumatic plasticity of corticospinal neurons in rats. Exp. Neurol. 60: 557-569.

5. Fowler, H., S. P. Hicks, C. J. D Amato, And F. A. Beach. 1962. Effects of fetal irradiation on behavior in the albino rat. J. Comp. Physiol. Psychol. 55: 309-314.

6. Hicks, S. P. 1958. Radiation as an experimental tool in mammalian developmental neurology. Physiol. Rev. 38: 337-356.

7. Hicks, S. P., B. L. Brown, AND C. J. D'Amato. 1957. Regeneration and malformation in the nervous system, eye, and mesenchyme of the mammalian embryo after radiation injury. Am. J. Pathol. 33: 459-481.

8. Hicks, S. P., AND C. J. D'Amato. 1961. How to design and build abnormal brains using radiation during development. Pages 60-97 in W. S. Fields ANd M. M. Desmond, Eds., Disorders of the Developing Nervous System. Thomas, Springfield, Ill.

9. Hicks, S. P., AND C. J. D'AMATo. 1966. Effects of ionizing radiations on mammalian development. Pages 195-250 in D. H. M. Woollam, Ed., Advances in Teratology, Vol. 1. Logos Press, London.

10. Hicks, S. P., AND C. J. D' Amato. 1968. Cell migrations to the isocortex in the rat. Anat. Rec. 160: 619-634.

11. Hicks, S. P., AND C. J D'Amato. 1970. Visual and motor function after hemispherectomy in newborn and mature rats. Exp. Neurol. 29: 416-438.

12. Hicks, S. P., ANn C. J. D'AmATn. 1975. Motor-sensory cortex-corticospinal system and developing locomotion and placing in rats. Am. J. Anat. 143: 1-42.

13. Hicks, S. P., AND C. J. D'AMATO. 1976. Labeling corticospinal and brain-stem neurons in mature, infant, and radiation-malformed rats by retrograde axonal transport of horseradish peroxidase. Abstr. Proc. Annu. Meeting Mich. Chap. Soc. Neurosci., Ann Arbor, Mich.

14. Hicks, S. P., AND C. J. D’AmATo. 1977. Locating corticospinal neurons by retrograde axonal transport of horseradish peroxidase. Exp. Neurol. 56: 410-420.

15. Hicks, S. P., AND C. J. D'Amato. 1978. Effects of ionizing radiation on developing brain and behavior. Pages 35-72 in G. Gottlieb, Ed., Studies on the Development of Behavior and the Nervous System: Early Influences. Academic Press, New York.

16. Hicks, S. P., AND C. J. D'AMATO. 1979. Hopping locomotion in rats irradiated in utero. Soc. Neurosci. Abstr. 5: 116.

17. Hicks, S. P., AND C. J. D'AMATo. 1980. Development of the motor system: hopping rats produced by prenatal irradiation. Exp. Neurol. 70: 24-39.

18. Hicks, S. P., C. J. D'Amato, And J. L. Falk. 1962. Some effects of radiation on structural and behavioral development. Int. J. Neurol. 3: 535-548.

19. Hicks, S. P., C. J. D'Amato, AND D. L. Jof TES. 1962. The nature of the radiosensitive cells in the developing nervous system studied with tritiated thymidine. Pages 199-205 in B. Gross AND V. ZelenY, Eds., Effects of Ionizing Radiation on the Nervous System. IAEA, Vienna.

20. Hicks, S. P., C. J. D'Amato, S. J. Klein, L. L. Austin, and B. C. French. 1969. Effects of regional irradiation or ablation of the infant rat cerebellum on motor development. Pages 739-753 in M. R. Sikov and D. D. Mahlum, Eds., Radiation Biology of the Fetal and Juvenile Mammal, Proceedings of the Ninth Annual Hanford Biology Symposium. US AEC, Div. Tech. Inform. Ext., Oak Ridge, Tenn.

21. Hicks, S. P., C. J. D'Amato, AND M. J. LowE. 1959. The development of the mammalian nervous system. I. Malformations of the brain, especially the cerebral cortex, induced in rats by radiation. II. Some mechanisms of the malformations of the cortex.J. Comp. Neurol. 113: 435-469.

22. Hildebrand, M. 1976. Analysis of tetrapod gaits: general considerations and 
symmetrical gaits. Pages 203-236 in R. M. Hermann, S. Grillner, P. S. G. Stein, AND D. G. Stuart, Eds., Neural Control of Locomotion. Plenum Press, New York.

23. Hruska, R. E., ANd E. K. Selbergeld. 1979. Abnormal locomotion in rats after bilateral intrastriatal injection of kainic acid. Life Sci. 25: 181-194.

24. LiPTON, J. M. 1966. Locomotor behavior and neuromorphological anomalies in prenatally and postnatally irradiated rats. Radiat. Res. 28: 822-829.

25. Long, J. A., AND P. L. Burlingame. 1938. The development of the external form of the rat with observations on the origin of the extraembryonic coelom and foetal membranes. Univ. Calif. Publ. Zool. 43: 143-184.

26. Mullenix, P., S. Norton, and B. Culver. 1975. Locomotor damage in rats after $\mathrm{X}$-irradiation in utero. Exp. Neurol. 48: 310-324.

27. NieuwenhuYs, R. 1976. Aspects of the morphology of the striatum. Pages 1-17 in A. R. Cools, A. H. M. Lohman, AND J. H. L. VAN DEN BERCKEN, Eds., Psychobiology of the Striatum. North-Holland, Amsterdam. 